\title{
Reação de cultivares de soja à mela (Thanatephorus cucumeris) em campo em dois estádios de desenvolvimento das plantas
}

\author{
Kátia de Lima Nechet ${ }^{1}$; Bernardo de Almeida Halfeld-Vieira ${ }^{1}$; Vicente Gianluppi ${ }^{1}$; Maurício Conrado Meyer ${ }^{2}$
}

${ }^{1}$ Embrapa Roraima, CP 133, 69301-970, Boa Vista, RR. E-mail: katia@ cpafrr.embrapa.br, ${ }^{2}$ Embrapa Soja, CP 131, 65800-000, Balsas, MA. Autor para correspondência: Kátia de Lima Nechet. katia@cpafrr.embrapa.br Data de chegada: 21/11/2006. Aceito para publicação em: 02/04/2008

\section{RESUMO}

Nechet, K.L.; Halfeld-Vieira, B.A.; Gianluppi, V.; Meyer, M.C. Reação de cultivares de soja à mela (Thanatephorus cucumeris) em campo em dois estádios de desenvolvimento das plantas. Summa Phytopathologica, v.34, n.3, p.277-279, 2008

A mela, causada pelo fungo Thanatephorus cucumeris, é uma das principais doenças da cultura da soja no estado de Roraima. O objetivo deste trabalho foi avaliar a reação de 15 cultivares de soja à mela em condições de cerrado em Roraima. Os parâmetros avaliados foram a porcentagem de área foliar infectada (AFI) no estádio $\mathrm{R}_{{ }_{5.5}}$ e a porcentagem de vagens com sintoma de mela
(VM) no estádio $R_{6}$. Houve diferença significativa entre as cultivares testadas segundo o teste de Fisher LSD a $1 \%$ de probabilidade nas duas avaliações. A AFI variou de 1,5\% (cv. Padre) a $62 \%$ (cv.BR-36) e a VM de $8 \%$ (cv.UFV-9 e cv. Juçara) a $55 \%$ (cv. BR-36). A correlação entre as duas avaliações foi significativa e positiva.

Palavras-chave adicionais: Glycine max; Rhizoctonia solani; Amazônia

\section{ABSTRACT}

Nechet, K.L.; Halfeld-Vieira, B.A.; Gianluppi, V.; Meyer, M.C. Field reaction of soybean cultivars to rhizoctonia aerial blight (Thanatephorus cucumeris) at two growth stages. Summa Phytopathologica, v.34, n.3, p.277-279, 2008

Rhizoctonia aerial blight (RAB), caused by the fungus Thanatephorus cucumeris is one of the most damaging diseases of soybean at Roraima, Brazil. The objective of this study was to evaluate the reaction of 15 soybean cultivars to RAB in a cerrado (savannah) ecosystem in the state of Roraima. The percentage of foliar area infected (FAI) and the percentage of pods with disease symptoms
(WP) were evaluated on soybean plants at R. ${ }_{5.5}$ and R. $_{6}$ growth stages, respectively. There were significative differences among cultivars tested based on Fisher LSD test in both evaluations. The FAI ranged from $1.5 \%$ (cv. Padre) to $62 \%$ (cv. BR-36) and WP from $8 \%$ (cv. UFV-9 and cv. Juçara) to $55 \%$ (cv. BR-36). Significative and positive correlation was observed between the evaluations.

Additional keywords: Glycine max; Rhizoctonia solani; Amazon

A cultura da soja [Glycine max (L.) Merrill] vem sendo implantada no estado de Roraima nas áreas de cerrado e mata de transição. A safra ocorre de maio a setembro, coincidindo com o período chuvoso da região. No momento, Roraima permanece como o único estado brasileiro produtor de soja sem a ocorrência da ferrugem asiática (Phakopsora pachyrizi Syd. \& Syd) (4). Por outro lado, nas condições locais, uma das principais doenças da cultura no estado é a mela causada pelo fungo Thanatephorus cucumeris (Frank) Donk (anamorfo Rhizoctonia solani Kuhn). Esta doença é considerada limitante ao cultivo da soja na região amazônica $(5,6)$.

Em função dessa importância e da ausência de informações sobre a reação dos genótipos de soja à mela nas condições de Roraima, este trabalho teve como objetivo avaliar as cultivares de soja BRS Tracajá, BRS Pati, UFV-9, Embrapa 63 (Mirador), Jataí, FT-16, BRS Juçara, BRS Sambaíba, BRSMA Seridó RCH, Padre, Leflore, BRS 219 (Boa Vista), BR-36, IAC 8-2 e IAC-8 em relação à mela nas condições do cerrado de Roraima.

O ensaio foi conduzido de maio a agosto de 2003, no campo experimental Monte Cristo, da Embrapa Roraima, localizado em ecossistema de cerrado (Latossolo amarelo, textura arenosa), com média de precipitação pluviométrica mensal de $135 \mathrm{~mm}$. O delineamento experimental foi em blocos casualizados com quatro repetições, sendo cada repetição constituída por uma parcela contendo quatro linhas de $10 \mathrm{~m}$. Ao redor da área e entre as parcelas foi plantado feijão (Phaseolus vulgaris L. cv. Carioca) para aumentar a densidade de inóculo natural de $R$. solani na área experimental. Duas avaliações foram feitas na área útil de cada parcela (10 plantas centrais). A primeira no estádio R. ${ }_{5.5}$ (maioria das vagens entre 75 e $100 \%$ de granação) onde avaliou-se a porcentagem da área foliar infectada (A.F.I.) das plantas na parcela. A

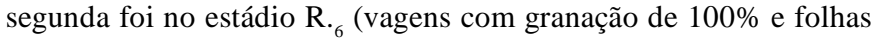
verdes), registrando-se o número total de vagens e o número de vagens com sintoma de mela para o cálculo da porcentagem de vagens com sintoma da doença (V.M.). Os dados foram submetidos à análise de variância no programa SAS versão 9 utilizando-se o proc GLM e o teste de Fisher LSD a $1 \%$ de probabilidade para a comparação de médias e o proc REG para cálculo do coeficiente de correlação de Pearson entra as variáveis A.F.I e V.M.

Todos as cultivares testadas apresentaram incidência da mela nas 
Tabela 1. Avaliação de 15 cultivares de soja (Glycine max) quanto à mela, causada pelo fungo Thanatephorus cucumeris, nas condições de cerrado do estado de Roraima, avaliando a porcentagem de área foliar infectada (AFI) no estádio R. ${ }_{55}$ e o percentual de vagens com sintoma da doença (V.M) no estádio R. 6 .

\begin{tabular}{|c|c|c|c|c|c|c|c|}
\hline \multirow{2}{*}{$\frac{\text { Cultivares }}{\text { UFV-9 }}$} & \multicolumn{3}{|c|}{$\operatorname{AFI}(\%)^{1}$} & \multicolumn{4}{|c|}{ V. $M(\%)^{1}$} \\
\hline & 2,00 & $\mathrm{a} \quad \mathrm{b}$ & & 8,1 & $\mathrm{a}$ & & \\
\hline BRS Juçara & 2,75 & $\mathrm{a} \quad \mathrm{b}$ & $\mathrm{c}$ & 8,2 & a & & \\
\hline BRS Tracajá & 1,60 & a & & 11,9 & $\mathrm{a}$ & & \\
\hline BRS Pati & 5,20 & $\mathrm{a} \quad \mathrm{b}$ & $\mathrm{c}$ & 11,9 & $\mathrm{a}$ & & \\
\hline BRS Sambaíba & 1,50 & a & & 13,9 & $\mathrm{a} \mathrm{b}$ & & \\
\hline Padre & 1,50 & a & & 14,7 & $\mathrm{a} \mathrm{b}$ & & \\
\hline BRS Seridó RCH & 1,60 & $\mathrm{a}$ & & 15,4 & $\mathrm{a} b$ & & \\
\hline BRS 219 (Boa Vista) & 2,00 & a $b$ & & 16,0 & $\mathrm{a} \mathrm{b}$ & & \\
\hline Embrapa 63 (Mirador) & 2,20 & $\mathrm{a} \quad \mathrm{b}$ & & 17,7 & $\mathrm{a} \mathrm{b}$ & & \\
\hline IAC $8-2$ & 4,60 & $\mathrm{a} \quad \mathrm{b}$ & $\mathrm{c}$ & 18,8 & $\mathrm{a} \mathrm{b}$ & & \\
\hline IAC-8 & 7,50 & & $\mathrm{c} d$ & 24,2 & $\mathrm{~b}$ & $\mathrm{c}$ & \\
\hline BRS Jataí & 8,20 & & $\mathrm{c}$ & 31,9 & & $\mathrm{c}$ & d \\
\hline FT-16 & 26,00 & & $\mathrm{~d}$ & 36,4 & & & $\mathrm{~d}$ \\
\hline Leflore & 22,50 & & $\mathrm{~d}$ & 54,1 & & & $\mathrm{e}$ \\
\hline BR-36 & 62,80 & & $\mathrm{e}$ & 54,8 & & & $\mathrm{e}$ \\
\hline
\end{tabular}

1 Médias seguidas pela mesma letra na coluna não diferem significativamente entre si pelo teste Fisher LSD a $1 \%$. folhas e nas vagens (Tabela 1).

Nas duas variáveis detectou-se a reação diferenciada das cultivares em relação à mela (Tabela 1). As cultivares com menor severidade da doença no estádio R. . $_{5.5}$ foram Padre (1,5\%), BRS Sambaíba (1,5\%), BRS Seridó $(1,6 \%)$ e BRS Tracajá $(1,6 \%)$ e com menor incidência de mela nas vagens no estádio R. ${ }_{6} \operatorname{UFV}-9(8,1 \%)$, BRS Juçara $(8,2 \%)$, BRS Tracajá $(11,9 \%)$ e BRS Pati $(11,9 \%)$. As cultivares BR-36, FT16 e Leflore apresentaram maior A.F.I. e incidência de mela nas vagens.

A correlação entre as duas variáveis de avaliação da doença foi significativa e positiva baseado no coeficiente de correlação de Pearson ( $\mathrm{p}<0,0001)$, ou seja, quanto maior a A.F.I. maior o número de vagens com sintoma da doença (Gráfico 1).

Dentre as cultivares testadas apenas BRS Sambaíba, BRS Pati, BRS Tracajá e BRS 219 (Boa Vista) são recomendados para plantio no estado de Roraima. A cultivar BRS Tracajá apresenta ciclo médio e as demais são precoces (2). Apesar da diferença significativa de incidência e severidade da mela entre os materiais, nas condições testadas, todas as cultivares recomendadas para Roraima apresentaram incidência baixa da doença em vagens (máximo de $16 \%$ para Boa Vista) e menor severidade (máximo de 5,2\% A.F.I. para BRS Pati). Nos parâmetros avaliados, destacou-se a cultivar BRS Tracajá, que é plantada em $90 \%$ das lavouras do estado.

A variabilidade de genótipos de soja para resistência à mela foi observada anteriormente por Meyer (6). Dentre 337 genótipos avaliados, 68,5\% foram considerados suscetíveis e apenas 3,9\%

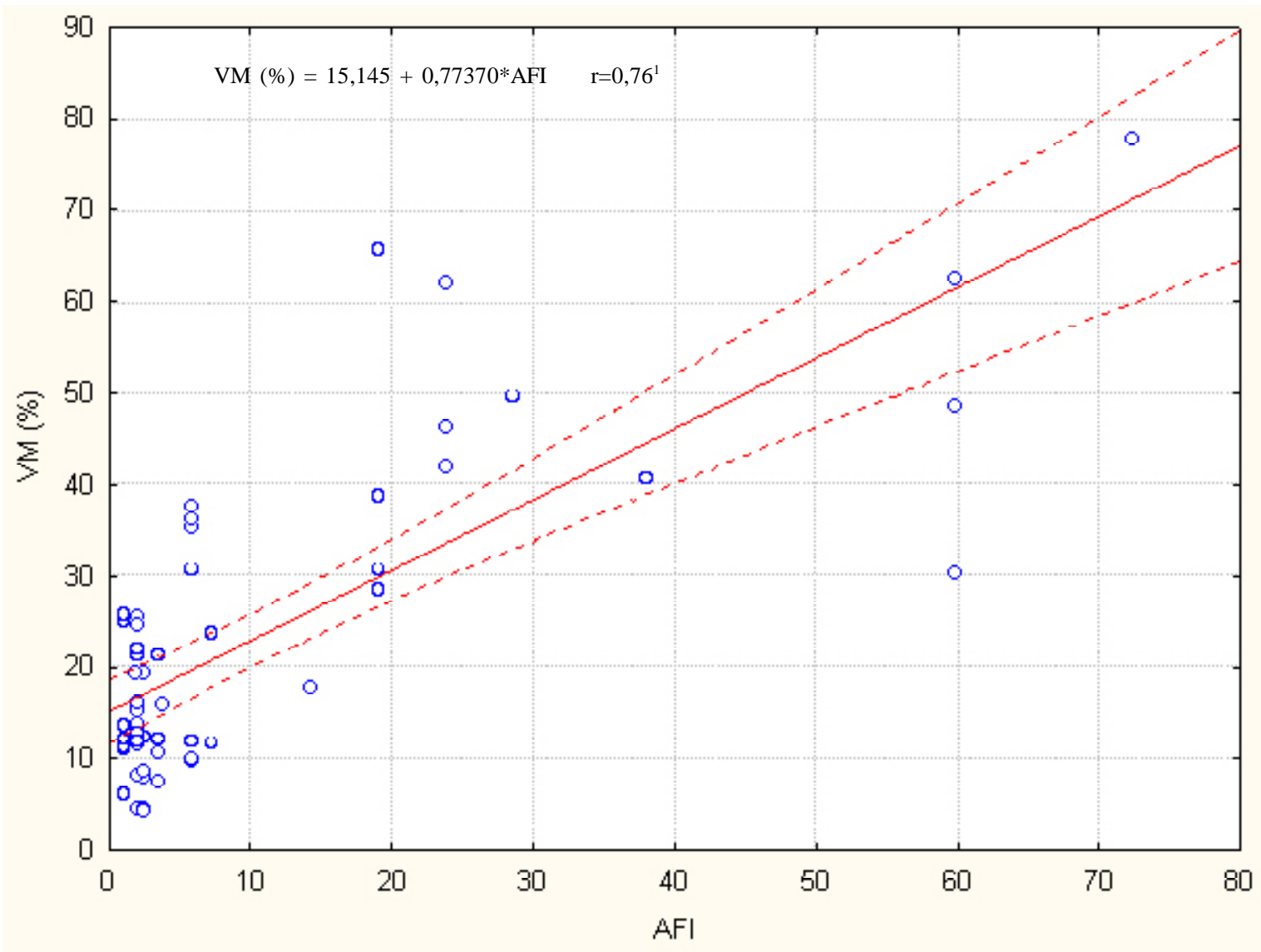

Gráfico 1. Relação entre a porcentagem de área foliar infectada (AFI \%) e o percentual de vagens com sintoma (VM \%) da doença. Os parâmetros AFI e VM foram obtidos da reação de 15 cultivares de soja (Glycine max) quanto à mela, causada pelo fungo Thanatephorus cucumeris, nas condições de cerrado do estado de Roraima.

${ }^{1}$ r ${ }^{3} 0,76$ - relação positiva e significativa entre os parâmetros avaliados. 
moderadamente resistentes. Outro aspecto importante que deve ser levado em consideração é a variabilidade genética de $R$. solani que é composta de pelo menos 12 grupos de anastomose (GAs) (7). O conceito de GAs de $R$. solani é uma ferramenta importante para os programas de melhoramento visando resistência. Neste trabalho não foi determinado o grupamento de anastomose prevalente no ensaio, porém estudos mostraram que a mela da soja é causada por $R$. solani AG1-IA nas regiões Central, Norte e Nordeste do Brasil, com exceção de Roraima, onde provavelmente é causada pelo AG1-IB $(1,3,6)$.

Os resultados obtidos neste ensaio indicaram que há reação diferenciada entre as cultivares quanto à mela e há genótipos promissores que podem ser trabalhados em programas de melhoramento genético visando resistência à doença. A correlação positiva entre A.F.I. e V.M. indica que ambos os parâmetros podem ser utilizados para diferenciar a reação de genótipos de soja à mela. Resultados similares foram observados por Stetina et al. (8). Portanto, nos trabalhos de melhoramento, onde se utiliza um número elevado de acessos, a avaliação da A.F.I. permite obter resultados em menos tempo e conduzir ensaios em condições de casa-de-vegetação.

Além disso, os resultados deste trabalho complementam as informações sobre a reação das cultivares recomendadas para Roraima quanto a doenças em ensaios conduzidos em outros estados. A reação de cultivares de soja à mela e o desenvolvimento de genótipos com características de tolerância e/ou de resistência à doença é essencial para a manutenção do sistema produtivo da cultura no estado de Roraima.

\section{REFERÊNCIAS BIBLIOGRÁFICAS}

1. Campos, A.P.S.; Ceresini, P.C. Incompatibilidade somática em Rhizoctonia solani AG-1 IA da soja. Summa Phytopathologica, Botucatu, v.32, n.3, p.247-254, 2006.

2. Cultivares de soja 2005: Regiões Norte e Nordeste. Documentos Embrapa Soja, Londrina, n.254, 2005. 28p.

3. Fenille, R.C.; Souza, N.L.; Kuramae, E.E. Characterization of Rhizoctonia solani associated with soybean in Brazil. European Journal of Plant Pathology, Wageningen, v.108, p.783-792, 2002.

4. Mattioni, J.A.M.; Souza, G.R.; Nechet, K.L.; Halfeld-Vieira, B.A Monitoramento de doenças da soja na safra 2006. Fitopatologia Brasileira, Lavras, v.32, supl., p.115-115, 2007. (Resumo).

5. Meyer, M.C.; Souza, N.L. Mela sob controle. Cultivar, Pelotas, n.58, p.26-29, 2004.

6. Meyer, M.C. Caracterização de Rhizoctonia solani Kühn, agente causal da mela da soja [Glycine max (L.) Merrill], seleção de genótipos e controle químico.2001. 125 f. Tese (Doutorado em Proteção de Plantas) - Faculdade de Ciências Agronômicas, Universidade Estadual Paulista, Botucatu.

7. Sneh, B.; Burpee, L.; Ogoshi, A. Identification of Rhizoctonia species. St. Paul: APS Press, 1991. 133p.

8. Stetina, K.C.; Stetina, S.R.; Russin, J.S. Comparison of severity assessment methods for predicting yield loss to rhizoctonia foliar blight in soybean. Plant Disease, St. Paul, v.90, p. 39-43, 2006 